\title{
A!
}

This is an electronic reprint of the original article.

This reprint may differ from the original in pagination and typographic detail.

Suhonen, P. M.; Linna, R. P.

\section{Dynamics of driven translocation of semiflexible polymers}

Published in:

Physical Review E

DOI:

10.1103/PhysRevE.97.062413

Published: 20/06/2018

Document Version

Publisher's PDF, also known as Version of record

Please cite the original version:

Suhonen, P. M., \& Linna, R. P. (2018). Dynamics of driven translocation of semiflexible polymers. Physical Review E, 97(6), [062413]. https://doi.org/10.1103/PhysRevE.97.062413

This material is protected by copyright and other intellectual property rights, and duplication or sale of all or part of any of the repository collections is not permitted, except that material may be duplicated by you for your research use or educational purposes in electronic or print form. You must obtain permission for any other use. Electronic or print copies may not be offered, whether for sale or otherwise to anyone who is not an authorised user. 


\title{
Dynamics of driven translocation of semiflexible polymers
}

\author{
P. M. Suhonen and R. P. Linna* \\ Department of Computer Science, Aalto University, P.O. Box 15400, FI-00076 Aalto, Finland
}

(Received 23 February 2018; published 20 June 2018)

\begin{abstract}
We study translocation of semiflexible polymers driven by force $f_{d}$ inside a nanometer-scale pore using our three-dimensional Langevin dynamics model. We show that the translocation time $\tau$ increases with increasing bending rigidity $\kappa$. Similarly, the exponent $\beta$ for the scaling of $\tau$ with polymer length $N, \tau \sim N^{\beta}$, increases with increasing $\kappa$ as well as with increasing $f_{d}$. By comparing waiting times between semiflexible and fully flexible polymers we show that for realistic $f_{d}$ translocation dynamics is to a large extent, but not completely, determined by the polymer's elastic length measured in number of Kuhn segments $N_{\text {Kuhn }}$. Unlike in driven translocation of flexible polymers, friction related to the polymer segment on the trans side has a considerable effect on the resulting dynamics. This friction is intermittently reduced by buckling of the polymer segment in the vicinity of the pore opening on the trans side. We show that in the experimentally relevant regime where viscosity is higher than in computer simulation models, the probability for this buckling increases with increasing $f_{d}$, giving rise to a larger contribution to the trans side friction at small $f_{d}$. Similarly to flexible polymers, we find significant center-of-mass diffusion of the cis side polymer segment which speeds up translocation. This effect is larger for smaller $f_{d}$. However, this speedup is smaller than the slowing down due to the trans side friction. At large enough $N_{\text {Kuhn }}$, the roles can be seen to be reversed, and the dynamics of flexible polymers can be reached. However, for example, polymers used in translocation experiments of DNA are elastically so short that the finite-length dynamics outlined here applies.
\end{abstract}

DOI: 10.1103/PhysRevE.97.062413

\section{INTRODUCTION}

Polymer translocation through a few nanometers wide pore has been studied extensively for two decades. The initial spur was given by the seminal paper by Kasianowicz et al. showing the potential of translocation in DNA sequencing [1]. Due to the potential applications and the originally missing theoretical framework, research has mostly concerned driven polymer translocation where force is exerted on the polymer segment residing inside the pore [2]. This force drives the polymer from the initial cis to the receiving trans side.

Most experiments on driven translocation are performed on semiflexible polymers, such as DNA. Despite this, all computer simulations in three dimensions until 2017 have been performed on translocation of fully flexible polymers. Also theoretically, fully flexible polymers have been of the highest interest. Very early Sakaue realized that the driven translocation dynamics is dominated by tension propagation in the polymer segment on the cis side [3]. Early computer simulations confirmed this showing that, during translocation, polymer segments on both sides of the membrane are driven out of equilibrium throughout the process [4]. An important addition was made by Rowghanian and Grosberg [5], and Sakaue together with Saito formulated the tension propagation theory to completion $[2,6,7]$. It is now established that driven translocation dynamics of fully flexible polymers is determined by tension propagation on the cis side where monomers sequentially join the dragged segment of the polymer increasing

*Corresponding author: riku.linna@aalto.fi the friction. Fluctuations, especially from the cis side, have been shown to aid the process [8-10]. The effect of monomer crowding on the trans side [4] has been shown to be negligible $[9,10]$.

After the general properties of driven translocation are understood via flexible polymers, the attention in computer simulations is shifting toward translocation of semiflexible polymers. Invoking the concept of Kuhn length, a semiflexible polymer in free space can be modeled at a lower length-scale resolution as a flexible chain. This approach is applicable if the polymer is long enough compared to its persistence length. The approach obviously fails when polymers are confined. Such a case is a polymer ejecting from a viral capsid. Here semiflexibility changes the dynamics of translocation fundamentally in a way that cannot be accounted for by a simple change in the length scale [11]. In driven translocation where polymer outside the pore is not confined it is an open question how well a change in length scale can account for changes brought in by polymer rigidity.

Driven translocation of semiflexible polymers has been studied by two-dimensional Langevin dynamics simulations $[12,13]$. Dynamics of translocating semiflexible polymers in two dimensions is expected to differ substantially from its three-dimensional counterpart. Three-dimensional simulations of semiflexible polymer translocation, accompanied by a simplifying model, have only recently been published [14]. Here the trans side friction was found to have a significant contribution on translocation time. Furthermore, the buckling of the polymer segment on the trans side was shown to reduce the friction and give it a characteristic dependence on the translocation coordinate $s$. However, the phenomenological 
characterization of buckling, which was found to be more probable for small than large pore force, leaves room for speculation.

Polymers used in experiments are relatively short compared to their persistence lengths. The longest polymers used in typical studies of Refs. [15-17] were only 323, 77, and 67 Kuhn segments in length, respectively. This suggests that finite-size effects, such as the intermittently buckling semirigid polymer segment on the trans side, may have a determining role in real-world translocation.

In what follows, we investigate driven semiflexible polymer translocation using our three-dimensional translocation model performing Langevin dynamics. On one hand, we set out to determine to what extent polymer rigidity can be accounted for by changing the relevant length scale. On the other hand, we will determine the effects of center-of-mass diffusion on the $c$ is side and the elastic buckling on the trans side on the dynamics of driven semiflexible polymer translocation. We describe the used computational models in Sec. II, present and analyze the obtained results in Sec. III, and make conclusions in Sec. IV.

\section{THE COMPUTATIONAL MODELS}

Here we describe the computational model for our threedimensional Langevin dynamics simulations. Except for the added bending potential, the present model is similar to what we have used in Refs. $[9,10]$. In addition, the pore model is slightly different from the one used in Ref. [9]. The simulation setup is depicted in Fig. 1.

\section{A. Polymer model}

We model the polymer as a wormlike chain consisting of a standard bead-spring model combined with a bending potential. Any two beads in the model repel each other via a truncated and shifted Lennard-Jones (LJ) potential given as

$$
U_{\mathrm{LJ}}= \begin{cases}4 \epsilon\left[\left(\frac{\sigma}{r}\right)^{12}-\left(\frac{\sigma}{r}\right)^{6}+\frac{1}{4}\right], & r \leqslant 2^{1 / 6} \sigma, \\ 0, & r>2^{1 / 6} \sigma,\end{cases}
$$

where $r$ is the distance between the two beads. $\epsilon=1$ and $\sigma=1$ define the units of energy and length in the model.

Two adjacent beads in the chain are connected via finitely extensible nonlinear elastic potential given as

$$
U_{\mathrm{F}}=-\frac{K}{2} R^{2} \ln \left(1-\frac{r^{2}}{R^{2}}\right), \quad r<R .
$$

$R=1.5 \sigma$ is the maximum elongation of the bond, and $K=$ $30 \sigma$ is the strength of the interaction.

The bending potential energy of the whole polymer is defined as

$$
U_{\text {bend }}=-\kappa \sum_{i}\left(\boldsymbol{r}_{i+1}-\boldsymbol{r}_{i}\right) \cdot\left(\boldsymbol{r}_{i}-\boldsymbol{r}_{i-1}\right) .
$$

Here, the sum runs over all beads $i$ in the polymer. $\boldsymbol{r}_{i}$ is the position vector of bead $i$, and $\kappa$ is the bending rigidity parameter. For $\kappa=0$, we have estimated from simulations that the persistence length $\lambda_{p} \approx 0.9$. This exceeds $\lambda_{p}=\frac{1}{2}$ (half bond length) due to excluded volume interactions. For higher $\kappa$ values, $\lambda_{p} \approx \kappa$. Polymer beads are indexed so that the bead

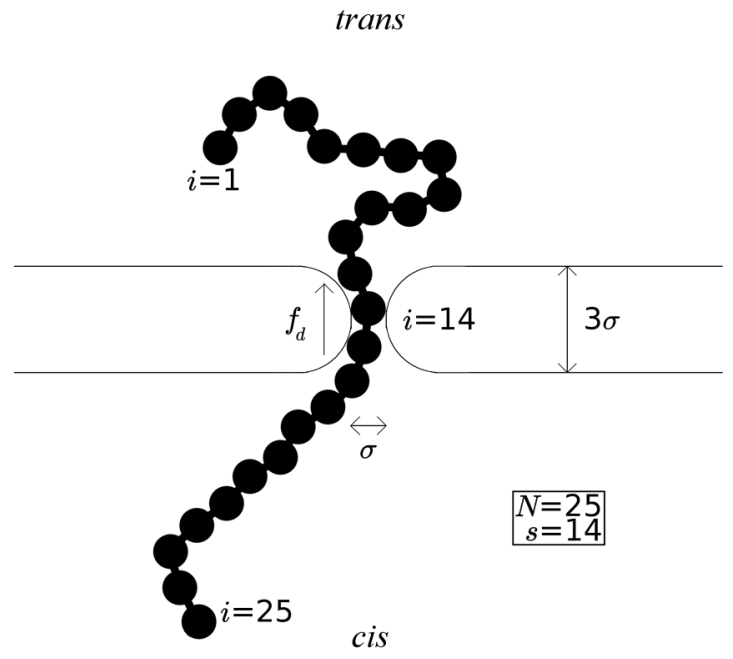

FIG. 1. A two-dimensional cross section of the three-dimensional simulation setup. A polymer chain of length $N=25$ is driven through a small pore in a membrane from the cis (bottom) to the trans (top) side. Driving force $f_{d}$ is applied on the polymer segment in the pore ( $f_{d}$ is divided among the beads currently occupying the pore). The membrane is $3 \sigma$ thick, and the pore is $1 \sigma$ wide at the narrowest point. Polymer beads are indexed as $i=1$ for the first bead to translocate and $i=N$ for the last bead to translocate. In the figure, 14 beads have crossed to the trans side, so the translocation coordinate is $s=14$. The bead radius used in the figure was chosen for clarity. The interaction distance between a monomer and a wall is measured from the center of the bead. The figure is reprinted with permission from Ref. [10] where aside from the bending potential, the simulation setup is the same.

of index $i=1$ translocates first and the bead of index $i=N$ translocates last.

\section{B. Simulation algorithm}

We use Ermak's implementation of Langevin dynamics $[18,19]$. Movement of all beads is determined by the Langevin equation,

$$
\dot{\mathbf{p}}_{i}=-\xi \mathbf{p}_{i}+\boldsymbol{\eta}_{i}(t)+\mathbf{f}_{i}\left(\mathbf{r}_{i}\right),
$$

where $\mathbf{p}_{i}$ is the momentum, $\xi=0.5$ is the friction coefficient, $\boldsymbol{\eta}_{i}$ is the Langevin random force, and $\mathbf{f}_{i}$ is the resultant of all other forces affecting the particle. For $\boldsymbol{\eta}_{i}(t)$ it holds that $\left\langle\boldsymbol{\eta}_{i}(t)\right\rangle=0$ and $\left\langle\boldsymbol{\eta}_{i}(t) \boldsymbol{\eta}\left(t^{\prime}\right)\right\rangle=2 \xi k T m \delta\left(t-t^{\prime}\right)$, where $\delta(t)$ is the Dirac $\delta$ function, $k$ is the Boltzmann constant, $T$ is the temperature, and $m$ is the mass of a bead. We set $k T=1$ and $m=16$. To integrate the equations of motion, we use the velocity Verlet algorithm [20]. For the integration, we set the time step $\delta t=0.001$ for translocation and $\delta t=0.025$ for equilibration prior to translocation.

\section{Pore and membrane models}

The geometrical aspects of the simulation setup are depicted in Fig. 1. We model the membrane as two impermeable planes at $r_{z}=-1.5 \sigma$ and $r_{z}=1.5 \sigma$. Points for which $r_{z}<-1.5 \sigma$ are on the cis side and points for which $r_{z}>1.5 \sigma$ are on the trans side. The pore is modeled as an opening around $r_{x}=r_{y}=0$. The opening has the shape of a hole in the 
middle of a torus. At the narrowest point the diameter of the pore is $\sigma$. The length of the pore is $3 \sigma$. The geometries are simulated using constructive solid geometry technique [21] applied to molecular dynamics simulations [22-24]. The membrane and pore geometries apply slip boundary conditions to beads colliding with the geometry. The pore is thus as generic as possible introducing no pore-polymer interactions other than those due to geometric constriction. The translocation is driven by force $f_{d}$ toward the positive $z$ axis. $f_{d}$ is divided among the beads inside the pore.

\section{The relation to real DNA molecules}

Reduced units are used in our simulations [19]. Here, we present a noncomprehensive mapping between our simulation space and the real world. We take our model polymers of $\kappa=20$ to represent double stranded DNA (dsDNA). The persistence length of dsDNA is around $50 \mathrm{~nm}$, corresponding to 150 base pairs (bps) [25-27]. For $\kappa=20, \lambda_{p} \approx 20 b$, where $b \approx \sigma$ is the bond length in our simulations. Now $\sigma=\frac{50 \mathrm{~nm}}{20} \approx$ $2.5 \mathrm{~nm}$. The thickness of the membrane is then $3 \sigma=7.5 \mathrm{~nm}$, which is comparable with the lipid bilayer thickness of $\approx 4 \mathrm{~nm}$ [28]. The shortest and longest polymers used in this article are $N=25$ and $N=400$. They correspond to the lengths $63 \mathrm{~nm}$ and $1 \mu \mathrm{m}$ (190 and $3000 \mathrm{bp})$, respectively. These lengths are comparable to dsDNA chains of 30-20 $000 \mathrm{bp}$ used in experiment of Ref. [29]. Each simulation monomer corresponds to $\frac{150 \mathrm{bp}}{20}=7.5 \mathrm{bp}=15$ nucleotides. The above mapping is only for the length scale. Relations for mass, energy, and time scales can also be calculated. As the mappings are not exact, we omit further treatment and refer the interested reader to Ref. [10] where we discuss the mapping in more detail in the case of flexible chains.

\section{RESULTS}

Here, we present the central results of our computer simulations on driven polymer translocation and discuss the characteristics pertinent to the bending rigidity of the simulated semiflexible polymers. The results are generally ensemble averages over about 500 simulations for each parameter set. For some parameters, more simulations were used for obtaining a better signal-to-noise ratio. In the initial conformations there are two beads on the trans side and three beads inside the pore. The rest of the beads are on the cis side. A bead is considered as translocated when it reaches the middle of the pore. Hence, at the start three beads are already considered translocated. These beads are subtracted from results involving the length of the polymer. Initially, before letting each polymer translocate it is equilibrated until its radius of gyration has converged to a stable value.

In Sec. III A, we report the measured waiting times and show how translocation dynamics changes due to bending rigidity. In Sec. IIIB, we show that the effects of bending rigidity are partially explained by polymer length in terms of Kuhn segments. In Sec. IIIC, we examine diffusive effects on the cis side. In Sec. IIID, we study buckling of the semiflexible polymer segment on the trans side. Lastly, in Sec. IIIE, we discuss the effect of bending rigidity on the scaling exponents of driven polymer translocation.
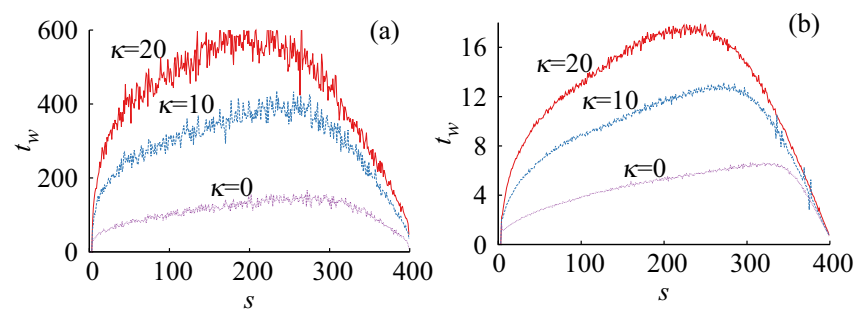

FIG. 2. Waiting times $t_{w}$ vs number of translocated monomers $s$. Polymer length $N=400$. Rigidity from bottom to top: $\kappa=0,10$, and 20. Pore force (a) $f_{d}=2$ and (b) $f_{d}=64$.

\section{A. Waiting times $t_{w}$}

We define waiting time $t_{w}(s)$ as the interval between instants that polymer beads (monomers) $s$ and $s-1$ enter the trans side for the first time. $t_{w}$ for polymers of length $N=400$ and rigidity $\kappa=0,10$, and 20 are shown in Fig. 2(a) for $f_{d}=2$ and in Fig. 2(b) for $f_{d}=64$. Polymer bending rigidity is seen to slow down translocation. Moreover, increasing $\kappa$ shifts the peak of the $t_{w}$ curve to smaller $s$, which means that tension propagation ends and retraction (also called postpropagation) starts earlier. In addition, for $f_{d}=2$ the peaks are more rounded and occur at smaller $s$ than for $f_{d}=64$. This is caused by diffusion of the polymer conformation on the cis side which we previously found out to be responsible for the same characteristic for flexible polymers [10]. The effect is stronger for smaller $f_{d}$ but can be seen in the strong $f_{d}$ regime to which all our simulations belong to.

Characteristics for different $N$ can be compared by plotting $t_{w}$ as a function of normalized reaction coordinate $s / N$. Figures 3(a) and 3(b) show $t_{w}(s / N)$ for fully flexible $(\kappa=0)$ polymers of lengths $N=25,50,100,200$, and 400 for $f_{d}=2$ and $f_{d}=64$. For shorter polymers $t_{w}$ peaks occur at smaller $s / N$.

The shift in the peaks of both Figs. 2 and 3 can be explained through the Kuhn length. Increasing $\kappa$ increases the Kuhn length, a statistical measure for polymer rigidity defined as the ratio of polymer's average squared radius and the maximum end-to-end distance $l_{\text {Kuhn }}=\left\langle R^{2}\right\rangle / R_{\max }$ [30]. The Kuhn length can be expressed as $l_{\text {Kuhn }}=n_{\text {Kuhn }} b$, where $n_{\text {Kuhn }}$ is the number of beads in a segment of length $l_{\text {Kuhn }}$ and $b$ is the equilibrium length of a polymer bond. A reasonable candidate for the effective length of a polymer, to take into account its rigidity, is the number of Kuhn segments $N_{\text {Kuhn }}=N / n_{\text {Kuhn }}$. Either increasing $\kappa$ for constant $N$ or reducing $N$ and keeping $\kappa$
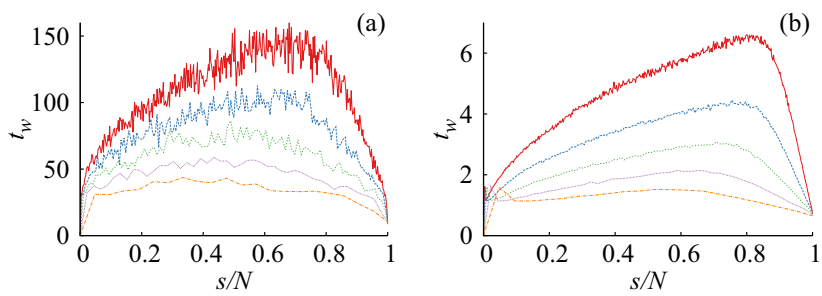

FIG. 3. Waiting times $t_{w}$ vs normalized reaction coordinate $s / N$ for fully flexible polymers $(\kappa=0)$. From top to bottom $N=400$, 200, 100, 50, and 25. Pore force (a) $f_{d}=2$ and (b) $f_{d}=64$. 

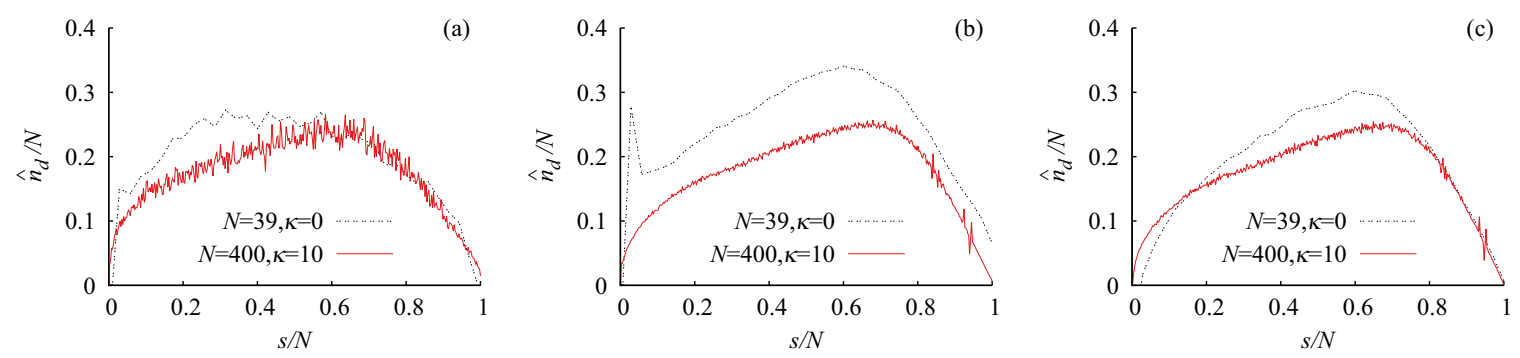

(c)
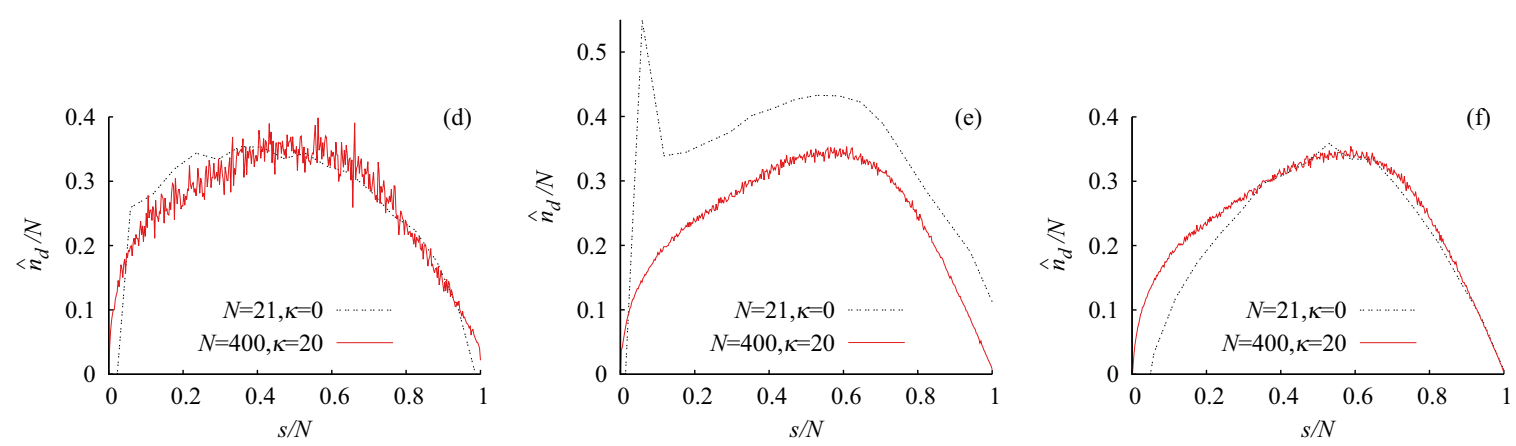

FIG. 4. The fraction of the total length of polymer under drag $\hat{n}_{d} / N$ as a function of normalized translocation coordinate $s / N$. Polymers of comparable lengths in the number of Kuhn segments are shown in each plot (see the text). The first row [(a)-(c)]: The first set of polymers has $N=39, \kappa=0$, and $N_{\text {Kuhn }}=20$. The second set of polymers has $N, \kappa=10$, and $N_{\text {Kuhn }} \approx 19.9$. The second row [(d)-(f)]: The first set of polymers has $N=21, \kappa=0$, and $N_{\text {Kuhn }} \approx 10$. The second set of polymers has $N=400, \kappa=20$, and $N_{\text {Kuhn }} \approx 9.9$. The first column [(a) and (d)]: $\xi=0.5, f_{d}=2$. The second column [(b) and (e)]: $\xi=0.5, f_{d}=64$. The third column [(c) and (f)]: $\xi=100, f_{d}=64$.

constant leads to shorter polymers in terms of $N_{\mathrm{Kuhn}}$. In accordance, maxima in $t_{w}$ are seen to shift toward smaller $s$ when increasing $\kappa$, Fig. 2, and smaller $s / N$ when reducing $N$, Fig. 3 . This trend is conceivable since in the extreme case of increasing $\kappa$ sufficiently so that $\lambda_{p}>N b$ the peak in $t_{w}$ disappears altogether and $t_{w}(s)$ becomes flat.

\section{B. Comparison of polymers of different $N$ and $\kappa$}

In free space and close to thermal equilibrium, semiflexible polymers of persistence length $\lambda_{p}$ can be modeled as fully flexible chains of bond length $b=2 \lambda_{p}$ by changing the degree of coarse graining $[31,32]$. In view of the findings of Sec. III A, it needs to be assessed to what extent bending rigidity can be taken into account in the context of driven translocation by merely adjusting the relevant length scale. We do this by estimating from simulations the ratio of the length of the polymer segment causing friction to the total polymer length $n_{d} / N . n_{d}=n_{d}^{c}+n_{d}^{t}+n_{d}^{p}$, where $n_{d}^{c}$ is the number of beads under drag on the cis side, $n_{d}^{t}$ is the number of beads currently being pushed on the trans side, and $n_{d}^{p}$ is the number of beads causing friction inside the pore.

In order to discuss the effective friction during translocation, we transform the measured waiting times to the number of monomers in drag. This transformation is performed conceptually in the overdamped limit and neglecting fluctuations where we have $\mathbf{f}_{i}\left(\mathbf{r}_{i}\right)=\xi \mathbf{p}_{i}$, see Eq. (4). Then $f_{d}=n_{d}(s) \xi m v(s)$, where $v(s)$ is taken as the average velocity of the $n_{d}$ beads at $s$. Waiting times $t_{w}(s)$ can be given in terms of velocity $v(s)$ and bond length $b$ as $t_{w}(s)=b / v(s)$. Since $b \approx \sigma$, we obtain $n_{d}(s)=\frac{t_{w}(s) f_{d}}{\xi m \sigma}$. The pore is $3 \sigma$ long, so approximately $n_{d}^{p}=3$ beads reside inside the pore throughout the translocation. To reduce the effect caused by the beads in the pore, which is relatively stronger for shorter polymers, we define $\hat{n}_{d}=n_{d}-n_{d}^{p}$. Since this transformation would be exact only in the overdamped limit with insignificant fluctuations, all effects, including those from diffusion, are included in $n_{d}$. This transformation of $t_{w}$ to $n_{d}$ is introduced in order to make the following discussion more clear.

Figure 4 shows $\hat{n}_{d} / N$ curves of a semiflexible and fully flexible polymers for $N$ and $\kappa$ chosen such that the polymer lengths in Kuhn segments $N_{\text {Kuhn }}$ are comparable. These lengths are calculated as $N_{\mathrm{Kuhn}}=(N-3) /\left(2 \lambda_{p}\right)$. Above, three beads are subtracted to account for the beads that are already translocated in the initial conformation of our translocation simulations. Semiflexible polymers of two different rigidities are compared with fully flexible polymers. In Figs. 4(a)-4(c) $\kappa=10$. In Figs. 4(d)-4(f) $\kappa=20$. The first column [(a) and (d)] and the second column [(b) and (e)] are for $f_{d}=2$ and 64 using friction $\xi=0.5$. In the third column [(c) and (f)] $f_{d}=64$ and $\xi=100$. The persistence length $\lambda_{p} \approx 0.9,10$, and 20 for $\kappa=0,10$, and 20 , respectively.

In Figs. 4(a) and 4(d) where $f_{d}=2, \hat{n}_{d} / N$ for flexible and semiflexible polymers of comparable $N_{\text {Kuhn }}$ are in general agreement. $\hat{n}_{d} / N$ for $s / N \lesssim 0.6$ is greater for fully flexible chains. These chains are very short in order to make them comparable to the semiflexible chains. The polymers are of thickness $\sigma$. Therefore, excluded volume interactions show more prominently for short polymers, and consequently the extension of polymers' initial conformation normalized with the polymer length is greater for short polymers. As translocation proceeds and the polymer extends, the excluded volume interactions decrease and vanish. In agreement with this $\hat{n}_{d} / N$ for short fully flexible polymers and semiflexible polymers are seen to align for $s / N \gtrsim 0.6$. 


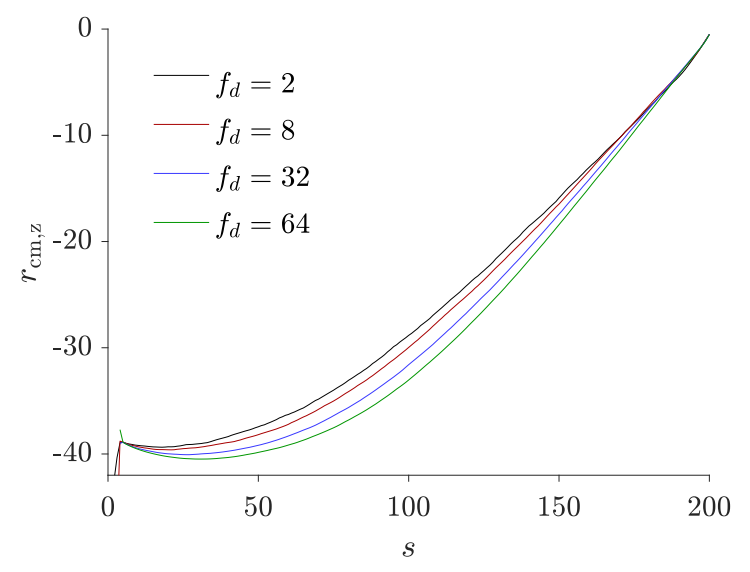

FIG. 5. The $z$ component of the center-of-mass position $r_{\mathrm{c} . \mathrm{m} . \mathrm{z}, \mathrm{of}}$ the cis side polymer segment. Measured for polymers of $N=200$ and $\kappa=20$. From top to bottom: $f_{d}=2,8,32$, and 64 . The pore center is located at $r_{\mathrm{c} . \mathrm{m} . \mathrm{z}}=0$.

As seen in Figs. 4(b) and 4(e) by increasing the bias to $f_{d}=$ 64 the agreement between flexible and semiflexible gets poorer. The short fully flexible chain shows a large (inertial) transient, which is to be expected at such a large bias. For large $f_{d}$, the pore friction is enhanced due to geometric effects local to the pore entrance $[9,10]$. This effect shows more prominently for short polymers since under identical viscous conditions friction outside the pore is larger for longer polymers. For any $s / N$ the same relative fraction of polymers is tensed and under drag for polymers of different $N$. The frictional contribution from the pore leads to overestimation of $\hat{n}_{d} / N$. This overestimation is larger for shorter polymers. Increasing viscosity outside the pore decreases the effect from the pore. This is seen in Figs. 4(c) and 4(f) where $\hat{n}_{d} / N$ are shown for $\xi=100$.

It is in place to note again that, in order to relate the length scale to polymer rigidity, we used the assumption of no fluctuations and strongly damped dynamics. Hence, all dynamics is reflected in the calculated friction or the number of beads in drag $n_{d}$. Clearly, for polymers of lengths used here there is a contribution on semiflexible polymers that cannot be accounted for by a simple length-scale transformation based on rigidity. To better determine the remaining contribution we next look at the contribution of diffusion in the next section.

\section{Diffusion on the cis side}

Previously, we observed clear $f_{d}$ dependent changes in the dynamics of driven translocation of fully flexible polymers. We showed these changes to be largely due to the center-of-mass diffusion of the polymer on the cis side [10]. Here, we show this center-of-mass diffusion to have a clear effect also on driven translocation dynamics of semiflexible polymers.

Figure 5 shows the $z$ coordinate of the center-of-mass position of the cis side polymer segment for $N=200$ and $\kappa=20$ polymers for various $f_{d}$. The position of the pore is at $r_{z}=0$. It is seen that for small $f_{d}$ the polymer's center of mass approaches the pore faster in $s$, i.e., faster with respect to the translocation time $\tau$. Without fluctuations, the center-of-mass movement as a function of $s$ would be similar regardless of $f_{d}$ [10]. Hence, the characteristics of Fig. 5 results

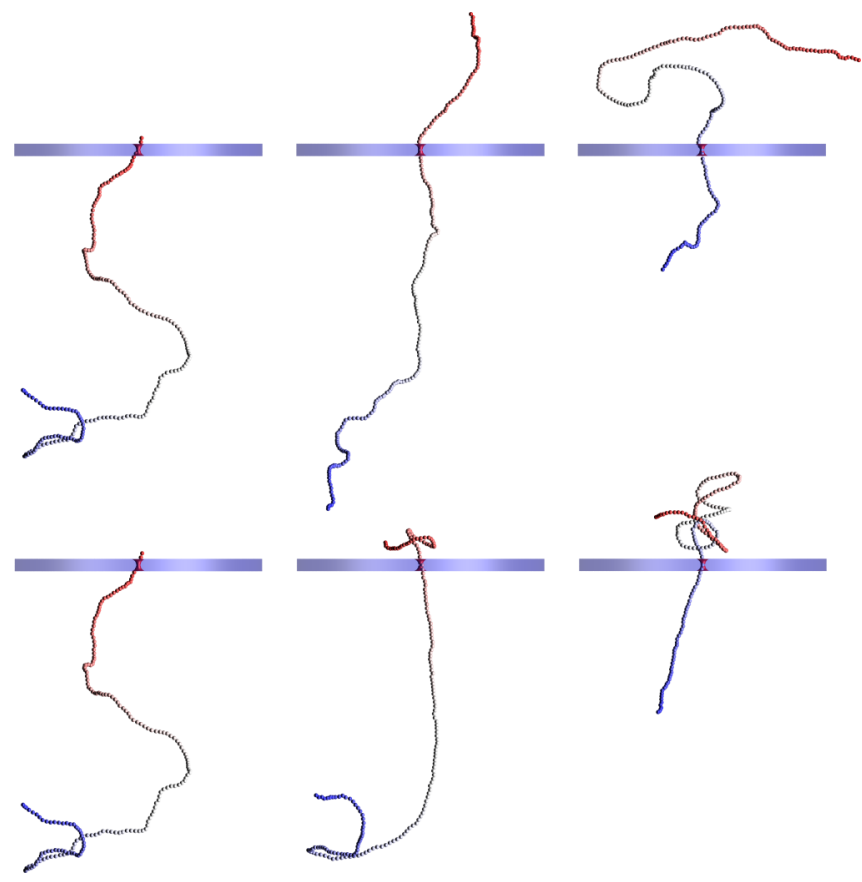

FIG. 6. Snapshots from simulations for $\kappa=20$ and $N=200$. Top row: $f_{d}=2$. Bottom row: $f_{d}=64$. From left to right snapshots are for translocation coordinates $s=4,50$, and 150. For smaller $f_{d}$ the polymer extends much farther on the trans side. The opposite is true for the tensed segment on the cis side where center-of-mass diffusion alleviates the tension for lower $f_{d}$.

from center-of-mass diffusion towards the pore. This confirms what was anticipated from indirect observations in Secs. III A and III B.

To summarize, the center-of-mass diffusion has a clear effect on both flexible and semiflexible polymer translocations. Making the length scales based on rigidity comparable in Sec. III B resulted in fair alignment of the driven translocation dynamics of flexible and semiflexible polymers for moderate $f_{d}$. As described, the diffusive effects were lumped into effective friction. The alignment would imply that taking into account the effective polymer length determined by polymer rigidity transforms also diffusive effects satisfactorily for moderate $f_{d}$.

\section{Trans side friction and buckling}

In contrast to fully flexible polymers, due to increased polymer chain rigidity, trans side friction can potentially be a significant factor in semiflexible chains of small $N_{\text {Kuhn }}$. In a recent study the effect of trans side friction and chain buckling characteristics were reported for one set of parameters and used in order to show agreement between a model and the experimental results on DNA translocation [14]. Here, we show that these characteristics are highly dependent, for example, on viscosity and pore bias $f_{d}$.

Figure 6 shows typical snapshots from our simulations of polymers of $N=200, \kappa=20$, and $f_{d}=2$ and 64 . Both of the presented simulations start from the same configuration. As the polymer translocates, the trans side segment first stays relatively straight and then bends and buckles. For higher $f_{d}$, 
(a)
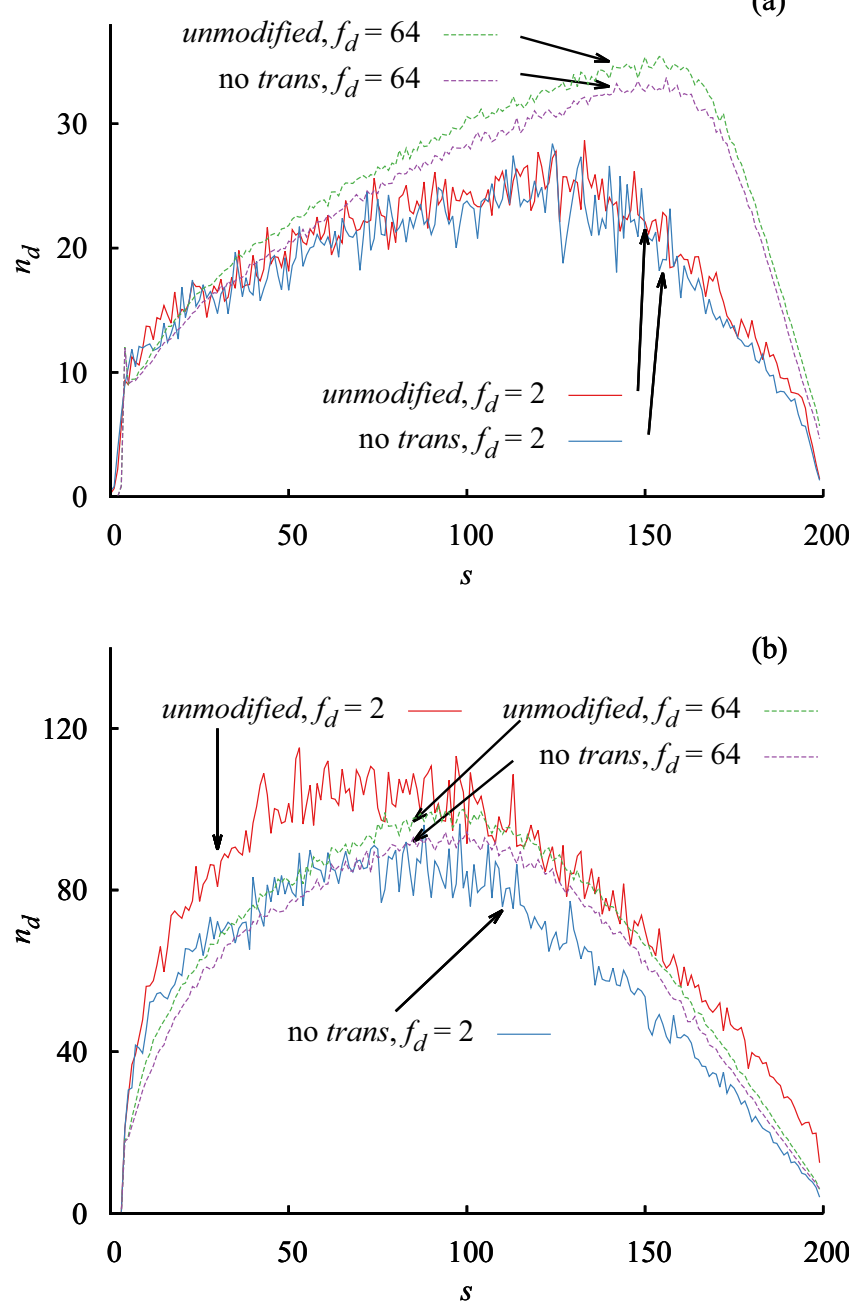

FIG. 7. Effective $n_{d}$ calculated from $t_{w}(s)$ for unmodified and no-trans translocation. $N=200 . f_{d}=2$ and 64. (a) $\kappa=0$ and (b) $\kappa=20$.

there is clearly a higher tendency for this buckling. So much so that the initially straight segment is barely visible for $f_{d}=64$.

Figure 6 indicates that the effect of trans side friction and buckling on the waiting times $t_{w}$ depends on $f_{d}$. We study these effects via $n_{d}$, the number of beads causing friction, defined assuming overdamping in Sec. III B as $n_{d}(s)=\left[t_{w}(s) f_{d}\right] /(\xi m \sigma)$. To pin down effects coming from the trans side, $n_{d}(s)$ for simulations where polymer beads are removed once they arrive at the trans side (denoted as "no trans") are compared with corresponding $n_{d}(s)$ for full polymers. We have used this bead removal previously for translocation of fully flexible polymers. Details of this procedure can be found in Refs. $[9,10]$.

In Fig. 7(a), $n_{d}(s)$ are shown for fully flexible $(\kappa=0)$ polymers of length $N=200 . n_{d}(s)$ for unmodified and notrans polymers are seen to be essentially identical. Hence, there is no significant trans side friction. Instead, the effect of diffusion can be seen clearly. The maximum $n_{d}(s)$ occurs at smaller $s$ for $f_{d}=2$ than for $f_{d}=64$. This is related to the center-of-mass diffusion of the cis side polymer segment [10]. The center-of-mass diffusion releases tension that is seen to grow farther in $s$ for $f_{d}=64$ since the role of diffusion is small for such an extreme bias. Also, since the tensed segment on the cis side is shorter for $f_{d}=2, n_{d}(s)$ is smaller for $f_{d}=2$ than for $f_{d}=64$.

Having found clear center-of-mass diffusion also for polymers of $\kappa=20$ in Sec. III C, we would expect $n_{d}$ curves for the semiflexible polymers to show similar dependence on $f_{d}$ as $n_{d}$ for fully flexible polymers. However, in Fig. 7(b) $n_{d}(s)$ for unmodified polymers of $\kappa=20$ is seen to be greater for $f_{d}=2$ than $f_{d}=64$. As seen in Fig. 6, the tensed segment on the cis side is longer for $f_{d}=64$, so this difference in semiflexible and fully flexible polymers can only originate from the trans side.

Moreover, the difference in $n_{d}$ between unmodified and notrans polymers, which is significant for $f_{d}=2$, is quite small for $f_{d}=64$. This implies higher trans side friction for smaller $f_{d}$. In Fig. 6 the trans side segment is seen to buckle earlier for higher $f_{d}$. This buckling reduces friction, so the earlier buckling for higher $f_{d}$ would explain the observed effect. This characteristics is at odds with findings by Sarabadani et al., who found that trans side segment buckles more easily for low $f_{d}[14]$.

For comparison, we look at trans side friction in the same way as was performed in Ref. [14]. In other words, we compute from our simulations the geometrical quantity $\tilde{\eta}_{T S}$ that was used as a measure of trans side friction in this paper. In order to calculate $\tilde{\eta}_{T S}$, a normalized angular cosine-correlation function is computed as $C(n)=$ $\cos \delta_{s-1} \cos \delta_{s-2} \cdots \cos \delta_{n} / \cos \delta_{s-1}$. Here angles $\delta_{i}$ from $i=$ $s-1$ to $n$ refer to angles of polymer bond vectors with the normal of the membrane. Index $i=s-1$ refers to the latest bond that has arrived at the trans side and $n \geqslant 1 . \tilde{\eta}_{T S}$ is calculated as a sum $\tilde{\eta}_{T S}=\sum_{i=s-1}^{n^{*}} \cos \delta_{i}$, where $n^{*}$ is defined as the smallest index $i$ for which $C(i)<1 / e$.

In Fig. 8 we show $\tilde{\eta}_{T S}$ for $N=200$ and $\kappa=20$ polymers with three different parameter sets. Figure 8(a) shows $\tilde{\eta}_{T S}$ for our simulation parameters where bead mass $m=16$, temperature $k T=1.0$, and friction coefficient $\xi=0.5 . \tilde{\eta}_{T S}$ is seen to be always lower for higher values of $f_{d}$. As the buckling reduces friction, this suggests easier buckling for higher $f_{d}$. For comparison, in Fig. 8(b) we use simulation parameters corresponding to those in Ref. [14]: $m=1, k T=1.2$, and $\xi=0.7$. At early stages of translocation, $\tilde{\eta}_{T S}$ is greater for higher $f_{d}$. After this, the same order as in Fig. 8(a) is resumed. Using these parameter values, we obtain qualitatively fairly similar characteristics for $\tilde{\eta}_{T S}$ as in Fig. 3 of the Supplemental Material of Ref. [14]. However, in Ref. [14], the curves of different $f_{d}$ converge to a same value as $s$ is increased. We do not observe this in either (a) or (b). Also, no sign of convergence is seen in Fig. 7(b) where the difference between unmodified and no-trans models stays constant for both $f_{d}=2$ and $f_{d}=64$. Possibly the used smaller length of the polymers explains the convergence observed in Ref. [14].

The proportion of dissipation to diffusion also affects the buckling. In translocation systems having weaker damping and higher diffusion, $\tilde{\eta}_{T S}$ can be higher for higher $f_{d}$. The dissipative term $-\xi m v_{i}$ in Eq. (4) is greater in Fig. 8(a) than in Fig. 8(b). We think that this causes the easier buckling for low $f_{d}$ at small $s$ in Fig. 8(b). It is noteworthy that also the bead mass is different. For easier comparison, in Fig. 8(c), $\tilde{\eta}_{T S}$ is shown for simulations where $\xi$ is one-tenth of $\xi$ in Fig. 8(a). The order 

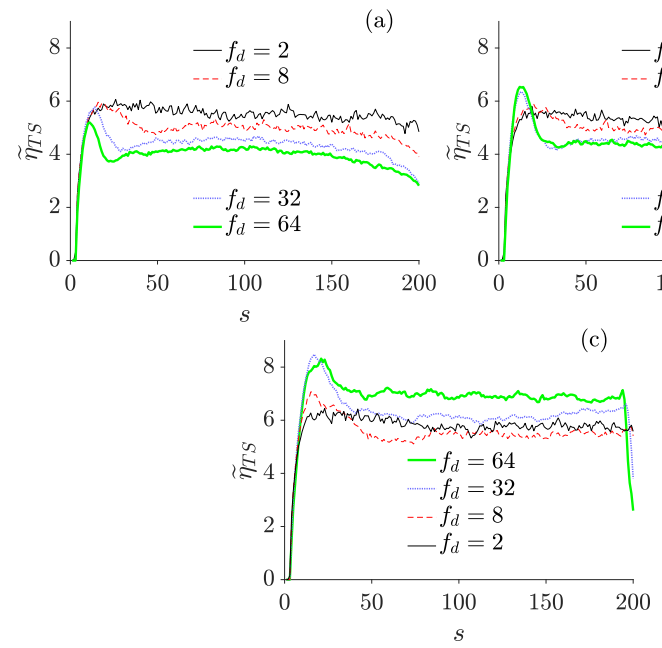

FIG. 8. Trans side friction contribution $\tilde{\eta}_{T S}$ calculated as in Ref. [14]. The figure corresponds to Fig. 3 of the Supplemental Material of Ref. [14]. For all (a)-(c): $\kappa=20$ and $N=200$. (a) $\tilde{\eta}_{T S}$ is calculated from simulations with same parameters as used in this article (parameters: $m=16, k T=1.0$, and $\xi=0.5$ ). (b) Except for $\kappa$ and $N$, we use simulations with the same parameters as in Ref. [14] (parameters: $m=1, k T=1.2$, and $\xi=0.7$ ). This effectively reduces the friction coefficient and increases the diffusion coefficient. (c) $\xi$ is decreased to one-tenth of what is used in (a) (parameters: $m=16, k T=1.0$, and $\xi=0.05$ ).

of the curves is clearly reversed, and buckling becomes easier for lower $f_{d}$.

Most computer simulation models are more diffusive and less dissipative than those encountered in nature [33]. As we argued in Ref. [10], the value of the friction coefficient should ideally be higher than those generally used in computational models. Although generally the exact friction coefficient value does not seem to be of substantial importance as long as the dynamics remains in the dissipative regime, in buckling it seems to have relevance. Intuitively one would expect buckling to take place more easily for higher $f_{d}$. Based on the presented results we expect this to be the case in the relevant experimental translocation systems of higher viscosity and lower diffusion.

To slightly elaborate on the buckling, a beam, on whose ends a force is exerted, buckles most likely in the middle. The probability for buckling also increases with the magnitude of the applied force. The situation is different for a semiflexible polymer chain being pushed from one end. Here, the drag force exerted on the chain is highest at the end where the force is exerted and decreases linearly along the chain. Hence, the drag force on the trans side is greatest close to the pore where the buckling is most likely to happen. Also, in this case the probability for buckling is higher for stronger force. The only factor that could change this is the thermal fluctuations of the heat bath. If the fluctuations are sufficiently strong compared to viscous drag force, then for a weak force the fluctuations deviating the polymer backbone from the axis passing through the center of the axis might cause the buckling to be more probable for a weaker force. However, this would require unrealistic low viscosity. In translocation the point at which the polymer segment on the trans side is pushed changes with
TABLE I. Scaling exponents $\beta$ for the power law fits $\tau \sim N^{\beta}$. Calculated from $N=50,100,200$, and 400 .

\begin{tabular}{lccc}
\hline \hline$f_{d}$ & $\kappa=0$ & $\kappa=10$ & $\kappa=20$ \\
\hline 2 & 1.42 & 1.49 & 1.52 \\
32 & 1.50 & 1.56 & 1.60 \\
64 & 1.49 & 1.56 & 1.61 \\
\hline \hline
\end{tabular}

$s$. The model of a rigid as yet unbuckled polymer being pushed at the end remains valid for all $s$.

\section{E. Scaling of translocation times $\tau$}

The average translocation time $\tau$ is known to obey the scaling $\tau \sim N^{\beta} f^{\alpha}$. In the very large $N$ and high $f_{d}$ regime $\beta=1+v$. For polymers of finite length and low $f_{d}, \beta<$ $1+v[4,5,34]$. On the other hand for a completely straight chain $\beta=2$ [35]. It is thus reasonable to expect that including a bending potential would increase $\beta(\leqslant 2)$.

In Table I the scaling exponent $\beta$ obtained from our simulations is shown for $\kappa=0,10$, and 20 and $f_{d}=2,32$, and 64. The results show the expected increase in $\beta$ as a function of $\kappa$. It is also seen that $\beta$ increases as a function of $f_{d}$ for all $\kappa$. This characteristic, found previously for fully flexible chains [4], evidently holds also for semiflexible chains.

The increase in $\beta$ as a function of $f_{d}$ is related to the center-of-mass diffusion in the low $f_{d}$ regime with fully flexible polymers [10]. The center-of-mass diffusion towards the pore alleviates tension, speeding up translocation. As Fig. 5 showed, this effect is present also for semiflexible polymers. For semiflexible chains there is also the $f_{d}$ dependent effect from trans side friction. Unlike diffusion, additional trans side friction slows down translocation for low $f_{d}$ thus reducing $\beta$. As seen in Table I the combined effect of diffusion and trans side friction results in an increase in $\beta$ with $f_{d}$.

The trans side friction also shows in the scaling exponent $\alpha$, see Table II. For fully flexible polymers, $\alpha>-1$, and for semiflexible polymers, $\alpha<-1$. This smaller $\alpha$ for semiflexible polymer translocation reflects the observed easier buckling of the trans side segment for stronger bias $f_{d}$ we found.

To compare the scaling of the translocation time for different $f_{d}$, we plot scaled translocation times $\tau f_{d}$ vs $N$ for $\kappa=0$ and $\kappa=20$ in Fig. 9. The range of polymer lengths $N \in[50,400]$ corresponds to $N_{\mathrm{Kuhn}} \in[28,222]$ for fully flexible polymers and $N_{\text {Kuhn }} \in[1.25,10]$ for semiflexible polymers. For any $N, \tau f_{d}$ increases with $f_{d}$ for flexible polymers and decreases for semiflexible polymers. The increasing trend for flexible polymers is due to the more prominent contribution of the center-of-mass diffusion on the cis side for small $f_{d}$. The change in this trend for semiflexible polymers shows that trans side friction contributes to the dynamics.

TABLE II. Scaling exponents $\alpha$ for the power law fits $\tau \sim f_{d}^{\alpha}$. Calculated from simulations with $N=200$ and $f_{d}=2,32$, and 64 .

\begin{tabular}{ccc}
\hline \hline$\kappa=0$ & $\kappa=10$ & $\kappa=20$ \\
\hline-0.92 & -1.02 & -1.04 \\
\hline \hline
\end{tabular}




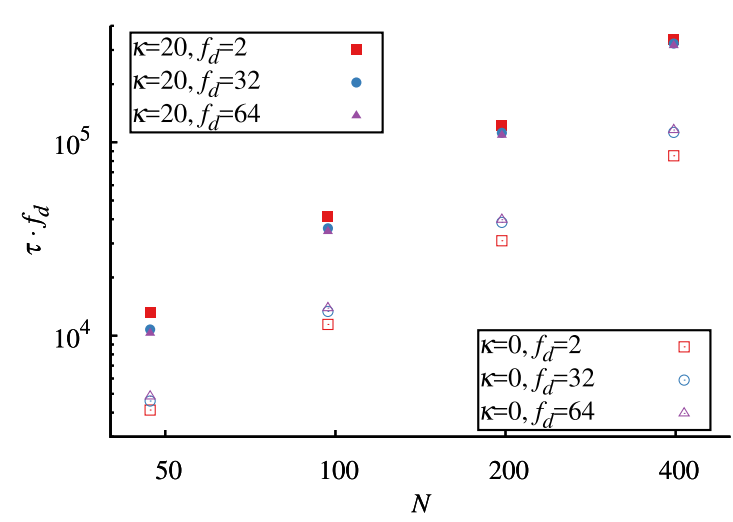

FIG. 9. Scaled translocation times $\tau f_{d}$ as a function of $N$. Lower set of points: $\kappa=0$, from the bottom up $f_{d}=2,32$, and 64 . The range of $N$ corresponds to $N_{\text {Kuhn }} \in[28,222]$. The upper set of points: $\kappa=$ 20, from top down $f_{d}=2,32$, and 64. The range of $N$ corresponds to $N_{\text {Kuhn }} \in[1.25,10]$.

For $\kappa=0$, the trend of $\tau f_{d}$ increasing with $f_{d}$ will be preserved for arbitrarily long polymers. When $\kappa=20$, the $\tau f_{d}$ curves for different $f_{d}$ will cross for sufficiently long polymers and will assume the same trend as the fully flexible polymers. This characteristics is reasonable. The trans side friction is more significant for small $N_{\text {Kuhn }}$. It is also more pronounced for low $f_{d}$ when buckling does not occur as easily as for high $f_{d}$.

The trans side friction and buckling dynamics are seen to be relevant for polymers that are short in terms of their persistence lengths. It should be noted that in fact DNA's of very finite lengths are used in experiments. For example, in experiments reported in Refs. [15-17] the longest dsDNA segments used were 97000,23000 , and 20000 bp corresponding to $N_{\text {Kuhn }} \approx$ 323,77 , and 67, respectively. In the recent paper by Carson et al. [29] the used dsDNA segments ranged from 35 to 20000 bp corresponding to $N_{\text {Kuhn }} \approx 0.12$ to $N_{\text {Kuhn }} \approx 67$. Sarabadani et al. fit their numerical model to this data [14]. The found scaling $\tau \sim N^{1.37}$ is far from the scaling $\tau \sim N^{1.6}$ expected for asymptotically long polymers as it should in the light of our findings. The dependence on $f_{d}$ is expected to be strong for the used polymer lengths. Consequently, in order to claim an agreement between the model and the experimentally found scaling, comparison should be made for at least two magnitudes of $f_{d}$. In addition, the model Sarabadani et al. use has no hydrodynamic interactions, which is generally known to have a strong effect on the scaling $\tau \sim N^{\beta}$, see, e.g., Refs. [4,36,37]. Accordingly, the model and the experiments in Ref. [14] should not even in theory be in agreement.

\section{CONCLUSION}

Here, we have studied driven translocation of semiflexible polymers through a nanoscale pore. We used Langevin dynamics simulations of a bead-spring polymer model including a bending potential characterized by rigidity parameter $\kappa$. The general characteristics of the process are aligned with expectations from physical considerations. Larger $\kappa$ resulted due to increased friction in slower translocation. Moreover, the scaling exponents $\beta$ in $\tau \sim N^{\beta}$ were found to increase as a function of $\kappa$, which is in keeping with $\beta \approx 1+v$ for fully flexible polymers and $\beta \approx 2$ for completely rigid polymers. In addition, $\beta$ was found to increase with $f_{d}$ for semiflexible polymers, similarly as has been found for fully flexible polymers [4].

To assess that driven translocation of semiflexible polymers shows unique characteristics not included in the respective process of fully flexible polymers, we made a comparison by introducing the polymer length measured in the number of Kuhn segments $N_{\text {Kuhn }}$. To make the discussion tangible, we somewhat simplistically transformed the waiting times to the fraction of polymers causing friction $\hat{n}_{d} / N$. We compared this quantity for fully flexible and semiflexible polymers, whose lengths were chosen such that the corresponding $N_{\text {Kuhn }}$ agreed as closely as possible. The agreement was found to be fair but not perfect especially for large $f_{d}$. Some of the differences can be attributed to differences in the excluded volume interactions and contribution from the effective pore friction, the magnitude of which is expected to change with changing $\kappa$. Even with these considerations, there clearly is a contribution from the trans side in driven semiflexible polymer translocation, which prevents treating semiflexible polymers as flexible polymers after a simple change in the relevant length scale for the used polymer lengths.

In contrast to fully flexible polymers where the trans side effect is small $[9,10]$, we found the increased correlations of semiflexible polymers to cause a significant additional trans side friction. We also found the intermittent buckling of semiflexible polymers to somewhat reduce this trans side friction, in accord with findings of Sarabadani et al. [14]. However, in contrast to those findings we observed the buckling to take place more easily at high $f_{d}$. This causes the relative contribution of trans side friction to be greater for smaller $f_{d}$ over the entire process again in contrast to Ref. [14] where higher $f_{d}$ results in an initially larger contribution, which then converges to the same friction for all $f_{d}$. This qualitative difference comes in one part from the greater dissipation present in our simulations and partly from polymers being longer in our Langevin simulations. The standardly used friction coefficients in computer simulations are generally small in comparison to real-world polymer systems [10]. This is especially true for the crowded environment inside cells. In general, as long as the dynamics remains in the overdamped regime, the qualitative results do not deviate. However, we find that the buckling dynamics of semiflexible polymers is more sensitive to changes in the system's dissipation. The easier buckling of the polymer at large $f_{d}$ is intuitively expected, and we showed that, by increasing a system's dissipation, and so bringing it closer to realistic systems, this characteristic is obtained. At a more general level, in addition to cis side diffusion, this trans side friction causes the scaling exponents to depend on the magnitude of the pore force.

We showed that, as in the case of fully flexible polymers [10], smaller $f_{d}$ results in significant center-of-mass diffusion toward the pore on the cis side. This speeds up translocation of flexible polymers by the alleviation of tension. For semiflexible polymers of low $N_{\text {Kuhn, }}$ this speedup is smaller than the slowing down due to the trans side friction. At some large enough $N_{\text {Kuhn }}$, the roles are reversed, and the situation becomes reminiscent to that of fully flexible polymers. However, DNA's 
in translocation experiments are quite short in terms of elastic rigidity. Hence, it is in fact dynamics of polymers of very finite lengths that is relevant.

Our simulations here do not take into account hydrodynamic interactions. Hydrodynamics speeds up driven translocation of flexible polymers and decreases $\beta[4,36,37]$. These effects are present also in the driven translocation of semiflexible polymers. Complete agreement of experimental measurements and computer models cannot be even theoretically expected if hydrodynamics is ignored and a comparison is made only using one pore force magnitude.

\section{ACKNOWLEDGMENTS}

P.M.S. thanks J. Piili for useful discussions. The computational resources of CSC-IT Centre for Science, Finland and the Aalto Science-IT Project are acknowledged. The work of P.M.S. was supported by The Emil Aaltonen Foundation.
[1] J. J. Kasianowicz, E. Brandin, D. Branton, and D. W. Deamer, Characterization of individual polynucleotide molecules using a membrane channel, Proc. Natl. Acad. Sci. U.S.A. 93, 13770 (1996).

[2] T. Sakaue, Dynamics of polymer translocation: A short review with an introduction of weakly-driven regime, Polymers 8, 424 (2016).

[3] T. Sakaue, Nonequilibrium dynamics of polymer translocation and straightening, Phys. Rev. E 76, 021803 (2007).

[4] V. V. Lehtola, R. P. Linna, and K. Kaski, Dynamics of forced biopolymer translocation, Europhys. Lett. 85, 58006 (2009).

[5] P. Rowghanian and A. Y. Grosberg, Force-driven polymer translocation through a nanopore: an old problem revisited, J. Phys. Chem. B 115, 14127 (2011).

[6] T. Saito and T. Sakaue, Dynamical diagram and scaling in polymer driven translocation, Eur. Phys. J. E 34, 135 (2011).

[7] T. Saito and T. Sakaue, Erratum to: Dynamical diagram and scaling in polymer driven translocation, Eur. Phys. J. E 35, 125 (2012).

[8] J. L. A. Dubbeldam, V. G. Rostiashvili, A. Milchev, and T. A. Vilgis, Driven translocation of a polymer: Fluctuations at work, Phys. Rev. E 87, 032147 (2013).

[9] P. M. Suhonen, K. Kaski, and R. P. Linna, Criteria for minimal model of driven polymer translocation, Phys. Rev. E 90, 042702 (2014).

[10] P. M. Suhonen, J. Piili, and R. P. Linna, Quantification of tension to explain bias dependence of driven polymer translocation dynamics, Phys. Rev. E 96, 062401 (2017).

[11] R. P. Linna, P. M. Suhonen, and J. Piili, Rigidity-induced scale invariance in polymer ejection from capsid, Phys. Rev. E 96, 052402 (2017).

[12] R. Adhikari and A. Bhattacharya, Driven translocation of a semi-flexible chain through a nanopore: A brownian dynamics simulation study in two dimensions, J. Chem. Phys. 138, 204909 (2013).

[13] A. Bhattacharya, Translocation dynamics of a semiflexible chain under a bias: Comparison with tension propagation theory, Polym. Sci., Ser. C 55, 60 (2013).

[14] J. Sarabadani, T. Ikonen, H. Mökkönen, T. Ala-Nissila, S. Carson, and M. Wanunu, Driven translocation of a semi-flexible polymer through a nanopore, Sci. Rep. 7, 7423 (2017).

[15] A. J. Storm, C. Storm, J. Chen, H. Zandbergen, J.-F. Joanny, and C. Dekker, Fast DNA translocation through a solid-state nanopore, Nano Lett. 5, 1193 (2005).

[16] D. Fologea, E. Brandin, J. Uplinger, D. Branton, and J. Li, Dna conformation and base number simultaneously determined in a nanopore, Electrophoresis 28, 3186 (2007).
[17] M. Wanunu, J. Sutin, B. McNally, A. Chow, and A. Meller, DNA translocation governed by interactions with solid-state nanopores, Biophys. J. 95, 4716 (2008).

[18] D. L. Ermak and H. Buckholz, Numerical integration of the Langevin equation: Monte Carlo simulation, J. Comput. Phys. 35, 169 (1980).

[19] M. P. Allen and D. J. Tildesley, Computer Simulation of Liquids (Clarendon, Oxford, 2006).

[20] W. F. van Gunsteren and H. J. C. Berendsen, Algorithms for macromolecular dynamics and constraint dynamics, Mol. Phys. 34, 1311 (1977).

[21] G. Wyvill and T. L. Kunii, A functional model for constructive solid geometry, The Visual Computer 1, 3 (1985).

[22] J. Piili and R. P. Linna, Polymer ejection from strong spherical confinement, Phys. Rev. E 92, 062715 (2015).

[23] J. Piili, P. M. Suhonen, and R. P. Linna, Uniform description of polymer ejection dynamics from capsid with and without hydrodynamics, Phys. Rev. E 95, 052418 (2017).

[24] J. Piili, Single Polymer Dynamics in Confined Geometries: Capsid Ejection and Sedimentation. Aalto University publication series, Doctoral dissertations, Espoo, 2017, http://urn.fi/URN:ISBN:978-952-60-7716-1.

[25] S. B. Smith, L. Finzi, and C. Bustamante, Direct mechanical measurements of the elasticity of single dna molecules by using magnetic beads, Science 258, 1122 (1992).

[26] G. S. Manning, The persistence length of dna is reached from the persistence length of its null isomer through an internal electrostatic stretching force, Biophys. J. 91, 3607 (2006).

[27] S. Geggier, A. Kotlyar, and A. Vologodskii, Temperature dependence of dna persistence length, Nucleic Acids Res. 39, 1419 (2011).

[28] K. Mitra, I. Ubarretxena-Belandia, T. Taguchi, G. Warren, and D. M. Engelman, Modulation of the bilayer thickness of exocytic pathway membranes by membrane proteins rather than cholesterol, Proc. Natl. Acad. Sci. U.S.A. 101, 4083 (2004).

[29] S. Carson, J. Wilson, A. Aksimentiev, and M. Wanunu, Smooth dna transport thrugh a narrowed pore geometry, Biophys. J. 107, 2381 (2014).

[30] M. Doi and S. F. Edwards, The Theory of Polymer Dynamics (Oxford University Press, New York, 1988).

[31] A. Y. Grosberg and A. R. Khokhlov, Statistical Physics of Macromolecules (American Institute of Physics, New York, 1994).

[32] M. Rubinstein and R. H. Colby, Polymer Physics (Oxford University Press, New York, 2016), reprint.

[33] H. W. de Haan, D. Sean, and G. W. Slater, Using a Péclet number for the translocation of a polymer through a nanopore to tune 
coarse-grained simulations to experimental conditions, Phys. Rev. E 91, 022601 (2015).

[34] T. Ikonen, A. Bhattacharya, T. Ala-Nissila, and W. Sung, Unifying model of driven polymer translocation, Phys. Rev. E 85, 051803 (2012).

[35] A. Y. Grosberg, S. Nechaev, M. Tamm, and O. Vasilyev, How Long Does It Take to Pull an Ideal Polymer into a Small Hole?, Phys. Rev. Lett. 96, 228105 (2006).
[36] M. Fyta, S. Melchionna, S. Succi, and E. Kaxiras, Hydrodynamic correlations in the translocation of a biopolymer through a nanopore: Theory and multiscale simulations, Phys. Rev. E 78, 036704 (2008).

[37] J. E. Moisio, J. Piili, and R. P. Linna, Driven polymer translocation in good and bad solvent: Effects of hydrodynamics and tension propagation, Phys. Rev. E 94, 022501 (2016). 